\title{
La place de l'histoire dans la pensée de Pareto
}

Bernard Valade

\section{(2) OpenEdition}

Journals

Édition électronique

URL : http://journals.openedition.org/ress/2535

DOI : $10.4000 /$ ress.2535

ISSN : 1663-4446

Éditeur

Librairie Droz

Édition imprimée

Date de publication : 15 décembre 2013

Pagination : 83-101

ISBN : 978-2-600-01805-0

ISSN : 0048-8046

Référence électronique

Bernard Valade, «La place de l'histoire dans la pensée de Pareto », Revue européenne des sciences sociales [En ligne], 51-2 | 2013, mis en ligne le 01 janvier 2017, consulté le 19 mars 2020. URL : http:// journals.openedition.org/ress/2535; DOI : https://doi.org/10.4000/ress.2535 


\title{
LA PLACE DE L'HISTOIRE \\ DANS LA PENSÉE DE PARETO*
}

BERNARD VALADE

Université Paris-Descartes

berval@paris5.sorbonne.fr

Résumé. De nombreux exemples que fournit le passé ont permis à Vilfredo Pareto de soutenir que l'histoire est «un cimetière d'aristocraties». Cependant, sa pensée sur I'histoire ne se réduit pas à cette affirmation. Dans ses ouvrages d'économie politique, comme dans son Traité de sociologie générale, il renvoie constamment aux travaux d'historiens modernes et contemporains. C'est à identifier les sources principales auxquelles il a puisé dans le domaine des études historiques, et à examiner l'usage souvent critique qu'il a pu en faire en sociologie, que le présent article est consacré.

Mots-clés: Vilfredo Pareto, école historique, historiographie, méthodologie, sociologie historique.

\begin{abstract}
Vilfredo Pareto based his statement that history is a "cimeterey of acristocraties" on many examples taken from the past. However, his thought on history is not to be reduced to this assertion. In his work on the political economy as well as in his Traité de sociologie générale, he constantly referes to the works of modern and contemporary historians. This article aims to identify the main sources he resorted to in the domain of historical studies, and to examine how he used them in an often critical way in sociology.
\end{abstract}

Keywords: Vilfredo Pareto, historical school, historiography, methodolgy, historical sociology.

* Je dédie cet article à Giovanni Busino. 
Les études rassemblées dans le dernier ouvrage consacré à Vilfredo Pareto (Femia, Marshall, 20I2) mettent l'accent, à juste titre, sur le caractère interdisciplinaire d'une œuvre qui intéresse autant l'économie politique que la sociologie générale. L'érudition historique dont sont lestés le Cours d'Économie politique I964 [I896I897]), Les Systèmes socialistes (1965b [190I-1902]), le Manuel d’Économie politique (1966b [1906]), et surtout le Traité de sociologie générale (1968 [1916]), n’y fait l'objet d’aucune mention. Elle ne fait certes pas du sociologue qui en fit grand usage un «historien». Spectateur, non engagé, des grands événements comme des vicissitudes de la vie politique de son temps qu'il observe et analyse, Pareto pourrait cependant faire figure de pionnier d'une «histoire immédiate»; les transformations de la démocratie, l'état de l'Europe après la Première Guerre mondiale et le «phénomène bolchevique» en fourniraient les principaux chapitres. Fréquemment formé d'emprunts aux travaux de nombreux historiens, le soubassement documentaire de ses quatre grands ouvrages oriente la recherche vers d'autres directions: l'identification des sources principales, leur emploi plus ou moins critique, les jugements portés sur leurs auteurs, la «méthode historique », l'«interprétation des faits». Du Cours au Traité, l'histoire ne cesse pas, chez Pareto, d'être sollicitée à titre de preuve ou d'illustration. Sans doute l'est-elle d’abord à l'appui de démonstrations économiques puis d'un raisonnement sociologique; mais le référentiel ne varie guère, et nombre d'orientations conceptuelles qu'il soutient sont d’emblée tracées.

\section{I.SAVOIR HISTORIQUE ET CONNAISSANCE SOCIOLOGIQUE}

Revenant en 1917, à l'occasion de son jubilé, sur son itinéraire intellectuel, Pareto (1975, p .65-70), a fortement souligné l'importance de l'histoire pour les sciences sociales. «Dans (celles-ci), l'expérience du passé se nomme histoire ; c'est donc dans l'histoire que nous trouvons un élément essentiel de ces sciences ; mais nous ne lui demandons pas seulement des descriptions, nous y cherchons aussi la connaissance des uniformités que présentent les phénomènes sociaux : elle nous renseigne sur les faits et sur les rapport des faits» (ibid., p. 67). En ce domaine, comme pour les autres, il s'est plu à citer les principaux ouvrages qui ont «plus ou moins agi » sur sa pensée : «en sociologie historique [l]es œuvres 
capitales, telles que celles de Fustel de Coulanges, de Sir Henry Sumner Maine, et les recherches de l'école historique allemande» (ibid., p. 69). On notera qu'il ne s'agit pas d'histoire «historisante» mais de «sociologie historique» en quête d’uniformités dont précisément «l'école dite historique » se désintéresse.

Que Pareto ait placé au premier rang l’auteur de La Cité antique (1864) n’a pas lieu de surprendre. Attaché avant tout aux «faits», Numa Fustel de Coulanges (I830-1889) professait que l'histoire ne se construit quavec des textes; il ne reconnaissait comme autorité que celle des Anciens, en ignorant délibérément les historiens modernes. Les thèses développées dans l'Histoire des institutions politiques de la France, dont le premier tome parut en i875, et au premier chef celles concernant les origines de la propriété foncière, devaient retenir l'attention de Pareto qui, par ailleurs, ne pouvait qu'être sensible à la démolition des illusions des révolutionnaires sur l'imitation des Anciens. Fustel est cité dans le premier volume du Cours notamment au paragraphe 559 à propos du système de propriété familiale, et dans le second tome au paragraphe 579. Ici, Pareto, après avoir précisé que «les recherches historiques sont d’un grand intérêt pour l'économie politique», fait le point sur les deux tendances qui les traversent : l'une qui reconstruit le passé à partir du présent, l'autre qui néglige complètement les «faits actuels». Pour lui, Fustel a réagi contre la première, en posant que l'historien doit exclusivement s'en tenir aux textes, mais «la sagacité avec laquelle il interprète les textes fait voir clairement qu’il sait tenir compte des faits du présent et des probabilités qui en sont la conséquence » (Pareto, 1964 [1897], \$579 n.I). L’autorité de Fustel est invoquée dans le premier tome des Systèmes socialistes, le Manuel, et le Traité. Dans celui-ci, elle est discutée, «à la marge » pourrait-on dire, aux paragraphes I028 et suivants. La contestation porte sur le caractère artificiel de l'origine des collectivités envisagées dans La Cité antique: son auteur «ne songe pas à démontrer que son raisonnement qui donne la religion comme l'“origine” de ces collectivités, n’est pas l'habituel post hoc propter hoc. C'est là justement le point faible de sa théorie». Se trouve en tout cas vérifié, et jusqu’à ces réserves, le jugement dont Pareto faisait part à 
Maffeo Pantaleoni (1984, lettre du 25 décembre I89I) à propos de Fustel de Coulanges : «La sua opera è importantissima $»^{1}$.

Toute aussi importante a été pour lui la contribution de Henry James Sumner Maine (I822-ı888) à l'anthropologie du droit. L'Étude sur l'histoire des institutions primitives (The Early History of Institutions, 1875) et les Études sur l'ancien droit publiées en I889 sont utilisées dans le Cours (op.cit., \$549 et §649); elles sont reprises dans Les Systèmes socialistes, où Maine est salué pour avoir observé, dans ses Essais sur le gouvernement populaire (I885, trad.I887), que «tout ce qui a rendu l'Angleterre célèbre, tout ce qui lui a donné la richesse a été l'œuvre de minorités parfois infimes » (1965b [190I-1902]), p. 107). L’Ancient Law (I86I) est citée deux fois dans le Manuel, particulièrement sur la question de la jurisprudence d'équité (op.cit., chap. VII, §ıo9). À ces différentes œuvres, le Traité renvoie une douzaine de fois dans les six premiers chapitres. L'intérêt que Pareto leur a porté tient à ce que leur auteur a révoqué les jugements de valeurs dont l'histoire du droit primitif des peuples européens était jusqu’alors encombrée.

Troisième source mentionnée en 1917, les « recherches de l'école historique allemande » ont exercé une influence diffuse dans l'œuvre parétienne. Ladite école peut être cependant entendue de différentes façons. Du point de vue de l'histoire économique, elle réunit deux générations. Disciple de Leopold Ranke, Wilhem Roscher (I8I7-I894) a travaillé sur des thèmes variés, de Thucydide à l'«économie nationale»; à cette mouvance appartiennent Karl Knies et Bruno Hildebrand. Ce ne sont pas ses Principes d'économie politique (1857) qui sont utilisés dans les deux volumes du Cours, mais des études spécialisées. De l'une d'elles est extrait un passage qui fait l'objet d'une note importante: selon Roscher, «les progrès de l'humanité ne s'accomplissent pas en ligne droite [...]

I On notera que les nombreuses œuvres citées de ce dernier le sont de façon plus ou moins précises. Les dates d'édition indiquées sont souvent celles de rééditions, et les titres parfois abrégés, sinon tronqués. Cette remarque vaut pour une bonne partie des sources mobilisées, singulièrement pour les auteurs étrangers: à la publication originale est substituée, la plupart du temps, la date de la traduction en français. Tantôt la citation est référencée avec un luxe de détails, en mentionnant, par exemple, le nom du traducteur; tantôt elle est donnée sans renvoi précis. Aux bibliographies irrégulièrement renseignées s'ajoutent des index lacunaires: n'y figurent pas tous les auteurs dont les travaux sont utilisés ou signalés. Exploitant à merveille des textes dont il avait besoin, Pareto ne s'est pas toujours soucié de les référencer exactement. 
mais suivent une sorte de spirale régulièrement interrompue, du moins en apparence, par des retours rétrogrades » (1964 [1896-1897], §927 n.3) ; c’est un fait dont il trouve la confirmation dans l'histoire de la science et qui donne lieu, chez Pareto, à des considérations sur la période ascendante et la période descendante. Mais Roscher est rangé parmi les économistes dans le discours du Jubilé. Gustav Schmoller (I838-1917), maître à penser de la Nouvelle École historique allemande, principal représentant de l'«Historische Schule der Nationalökonomie» fait figure, aux yeux de Pareto, de chef de file de la «Giovane scuola storica». Ses idées sur la solidarité, comme celles de Adolph Wagner, sont discutées dans un article de février i898 (1965a [1898], p. 266-276). Leur auteur est critiqué dans plusieurs lettres adressées à Pantaleoni : «E evidente che non sa l'economia politica » (1984, lettre du 19 janvier I898), et il n'est apparemment point fait grand cas de la «metodo storico degli Schmoller \& Cie» (ibid., lettre du 2 avril 1907). On ne relève qu’une seule occurrence dans les œuvres majeures (Les Systèmes socialistes, op.cit., p. 99), à propos du débat sur le socialisme de la chaire où les positions de Julius Wolf sont plus attentivement examinées.

Sur le plan de l'historiographie générale, le renouvellement des études manifesté en Allemagne par la fondation, en I859, de la revue Historische Zeitschrift, non plus que l'œuvre de Ranke (I795-1886), n’ont d'écho dans la sociologie de Pareto. Ce dernier, particulièrement attaché aux «faits», aurait pu être sensible à la définition de l'histoire comme discipline heuristique, et à la conception du travail de l'historien qui doit se vouer à la recherche de l'objectivité et retracer la manière dont le passé s'est réellement déroulé («bloss zeigen, wie es eigentlich gewesen »). Mais il était davantage intéressé par l’intégration de ces faits dans une vision de l'histoire nourrie d'emprunts à divers domaines disciplinaires. D'où ses renvois fréquents à la Römische Geschichte (I8II) de Bartold Georg Niebuhr et surtout aux ouvrages de Theodor Mommsen (I8I7-I903). Parmi ceux-ci, la Römische Geschichte (3 vols I854-1856; t.V, 1885, le t. IV n’a jamais paru) se présente comme une histoire totale de l'antiquité romaine où sont intégrés les apports de multiples sciences: épigraphie, philologie, archéologie, jurisprudence, etc.). À des travaux spécialisés du même historien, sur le droit public et le droit pénal romain, renvoie également et abondamment le Traité. 
Ces contributions majeures à la «sociologie historique» ne sont pas exclusives d'autres sources toutes aussi importantes. Le tableau de la cité antique brossé par Fustel de Coulanges trouve son pendant dans celui qu’a donné Tocqueville de la société moderne. Dans le Cours, le premier renvoi (non indexé) à l'auteur de L’Ancien Régime et la Révolution (1856) se trouve en bas de la note 6 du paragraphe 363 consacrée au jugement porté par Taine sur l'émission des assignats : «Tocqueville a beaucoup mieux jugé les faits, quand il a observé que la Révolution n’a fait qu'employer les procédés de l’ancien régime». Après une longue citation du même livre (1964 [I896-I897], §637 n.I), Pareto y revient (ibid., §799 n.I) pour reproduire la même remarque; on la retrouve mots pour mots dans Les Systèmes socialistes (op.cit., p. I34) où Tocqueville est salué pour avoir « décrit fort bien la période d’oscillation irréligieuse qui précéda la Révolution française» (ibid., p. 32), en des «lignes (qui) paraissent avoir été écrites pour la France de nos jours» (ibid., p. 33). Les analyses de Tocqueville sont encore discutées dans les dernières pages du Traité (op.cit., §2566 n.I) avec celles de Taine. On sait en quelle estime Pareto tenait la pensée de Tocqueville-dont il ne cite jamais De la Démocratie en Amérique. À Pantaleoni, il écrivait: «Il Tocqueville è immensamente superiore al Taine. (Il) a veduto che la rivoluzione non aveva fa altre che adoperare le armi delle quali si serviva la monarchia assoluta» (1984, lettre du 22 décembre I89I); et au même, quelques années plus tard, «io non conosco storie moderne scientifiche, salvo forse il Tocqueville» (ibid., lettre du 8 décembre i898). Pour Pareto, en effet, la fresque des Origines de la France contemporaine (I875-1893) n’a jamais fait figure de modèle. ${ }^{2}$

En histoire du droit, les conceptions de H. S. Maine sont souvent comparées chez Pareto à celles de Rudolph Jhering (I8I8-I892). De longs extraits de la traduction française de Geist des römischen Rechts (1852-I860) -L'esprit du droit romain dans les différentes phases de son développement (4 vols, I880)-, où le droit est traité comme un organisme vivant, détaché de tous les particularismes et revêtu d'une signification objective («intérêt juridiquement protégé»), sont donnés à plusieurs reprises dans le Traité. Au paragraphe 24I, il est estimé que «Ihering serre de plus près que Maine » un point précis de la science juridique romaine. 
Dans Les Systèmes socialistes, il est cependant reproché à l'auteur de Zweck im Recht (I877-I883) de ne pas s'en tenir à la matière qu'il «sait à fond», et de se mêler de questions touchant à l'économie politique qu'il ignore. En effet, sa conception de la justice est «malheureusement accompagnée de développements métaphysiques fort peu satisfaisants et de propositions vagues et sans réalités concrètes » (Pareto, 1965b [1902], p. I14).

Les «recherches del'école historique allemande» ne constituent enfin qu'une petite partie de la documentation placée chez Pareto sous le signe de Clio. Pour l'Antiquité, il a fréquenté assidûment les neuf fascicules du Dictionnaire des antiquités grecques et romaines (1873-I884) de Charles-Victor Daremberg et Edmond Saglio, les sept volumes du Handbuch der römischen Alterthümer (I87I-I885) de Joachim Marquardt et Theodor Mommsen, les travaux, parmi les plus notables, de Gaston Boissier, Auguste Bouché-Leclercq, Victor Duruy, Gustav Glotz. Pour chaque période ou chaque sujet, il puise aux meilleures sources : Jacob Burckhardt sur la Renaissance en Italie, Jacques Flach sur les origines de l'ancienne France, Johannes Janssen sur l’Allemagne et la Réforme, Siméon Luce sur la jacquerie. La collection des Mémoires relatifs à l'histoire de France dirigée par François Guizot lui est familière, comme les œuvres d'historiens renommés-Augustin Thierry, Jules Michelet par exemple-ou de savants austères tel Émile Levasseur (I828-I9II)-, un des fondateurs, injustement oublié, de l'histoire économique et sociale en France. S’il ne cite qu'exceptionnellement les deux grands historiens du XVII siècle qu'on été JeanBaptiste Vico et Edward Gibbon, il tire ses renseignements sur l'histoire de l'Amérique de William Robertson (I72I-1793). Il a beaucoup utilisé, dans leurs éditions françaises, l'Histoire du développement intellectuel de l'Europe (I868) de John William Draper, les 19 volumes de l'Histoire de la Grèce (I864-I867) de George Grote, l'Histoire de la civilisation en Angleterre (1865) de Henry Thomas Buckle. Plusieurs pièces de sa correspondance ${ }^{3}$ montrent l'admiration qu'il professait à l'endroit de ce dernier. Dans le Cours (op.cit., §687, n.I) est ainsi célébré «l’admirable tableau que donne Buckle des maux causés par l'ignorance et la superstition en Espagne», compliment assez rare de la part d’un savant qui, plus souvent sévère qu'élogieux, en était fort avare.

3 Voir notamment la note de Gabriel de Rosa qui accompagne la lettre à Pantaleoni en date du 4 août 1892 (Pareto, 1984, p. 262). 
Ce jugement sur le modèle d'histoire scientifique que constitue pour Pareto, selon Gabriel de Rosa (Pareto, 1984, p. 334), l'Histoire de Buckle, fait contraste (voir Cours, op.cit., \$672 n.I) avec celui que lui inspire «l'école économique dite historique ». Sont ici inégalement visés deux des fondateurs anglais de l’histoire économique, James Edwin Thorold Rogers (I823-I890) et William James Ashley (1860-1927). Les faits enregistrés par le premier «dans ses volumes sur L’Agriculture et les prix au Moyen Age» (Pareto, 1965b [1902], p. 299)-il s'agit de l'History of Agriculture and Prices in England from I259 to I793 dont le premier volume paraît en I866-, sont dûment commentés : «l'auteur a étudié à fond la question». Son Interprétation économique de l'histoire (I888), objet de plusieurs renvois, a été moins bien traitée: Thorold Rogers ne s'en tient pas, comme il le prétend, à l'observation, il «donne des préceptes [...] En général, dans les livres de l'école "historique", la partie descriptive est bonne [...]. Au contraire, la partie déductive, surtout quand elle veut s'élever aux principes généraux, est parfois extrêmement faible» (Pareto, 1964 [1896-1897], §577). Ashley, quant à lui, est un de ces théoriciens de «l'école soi-disant historique (qui) ont un parti pris de tout justifier dans le passé » (Les Systèmes socialistes, op.cit., p. 200 et n.I). Ils ne savent rien des faits et de leurs rapports dont ils veulent faire l'histoire. L'auteur d'An Introduction to English Economic History and Theory (1888-1893), est encore ironiquement pris à partie quelques pages plus loin (ibid., p. 209). Arnold Toynbee (1852-1883), une des figures majeures de cette école, est délibérément ignoré : ses fameuses Lectures sur la révolution industrielle faisaient aux "idées" une place beaucoup plus grande quaux "faits".

La «prétendue “école historique” » est maintes fois stigmatisée dans l'œuvre parétienne. Ses membres ne tiennent pas seulement compte des faits : ils se donnent comme «révélateurs » de lois de l'histoire. Auguste Comte a été, à cet égard, «le précurseur de plusieurs auteurs » de cette prétendue école (ibid.) aimantée par l’idée de prévision. Mais Pareto ne cessera de le répéter: les «prétendues lois historiques » sont sans valeur : «C’est parce que le mouvement social a lieu selon une courbe ondulée, qu’il est difficile de prévoir, d’après les faits du passé, le sens futur de ce mouvement» (ibid., p. 3 I). «Les phénomènes sociaux présentent, en général, une marche ondulée. Il y a des périodes où l’in- 
tensité croît, d’autres où elle décroît ; Il est bien difficile de savoir si la période d'accroissement observée jusqu’à présent ne va pas, tôt ou tard, être suivie d'une période de décroissement » (ibid., p. 344).C'est là un thème qui est introduit, comme il est rappelé en note, dans le Cours (op.cit., §578).

Parmi les recensions d'ouvrages réunies par Giovanni Busino dans Mythes et idéologies (1966a), six comptes rendus attestent l'intérêt de Pareto pour l'histoire économique et sociale. Tous ont été publiés, entre I898 et I90I, dans Zeitschrift für Socialwissenschaft. Ils sont l'occasion de commentaires, plus ou moins développés, appelés à être ultérieurement repris, dans le Traité notamment. Le jugement porté sur l'Histoire des corporations de métiers de Étienne Martin Saint-Léon est sévère : le chapitre consacré aux économistes «contient presque autant d'erreurs que de mots » (ibid., p. I3I); il est mesuré pour Influenza del cristianismo sulla economia de Giovanni Lerda : beaucoup de réserves sont à faire, mais «l'érudition est de bon aloi » (ibid., p. I39) ; très positif concernant le livre de Henri Hauser, Ouvriers du temps passé ( $\mathrm{x} v^{e}$ et $\mathrm{xvI}$ siècle) qui «jette un jour nouveau sur bien des questions se rapportant à l'histoire du travail en France » (ibid., p. I8I) ; La main-d'œuvre industrielle dans l'ancienne Grèce de Paul Guiraud est un «excellent ouvrage» (ibid., p. 192) où se trouvent confirmées des observations présentées dans le second volume du Cours; le quatrième tome de l'Étude historique sur les corporations professionnelles chez les Romains de Jean Pierre Waltzing «deviendra classique» : tout ce qui y est exposé s’appuie «sur de nombreux faits judicieusement interprétés » (ibid., p. 199).

On retrouve dans le Traité le nom de Waltzing, de même que celui de Ettore Ciccotti dont Il tramonto della schiavitù nel mondo antico fait l'objet de la première recension dans l'ordre chronologique; celle-ci est la plus importante, de par la personnalité de l'auteur', et en ce qu'elle introduit des considérations (ibid, p. I25-I29) sur la causalité en histoire qui seront par la suite enrichies. À cet ensemble d'analyses, il faut adjoindre celle de l'Histoire politique de l'Europe contemporaine de Charles Seignobos; elle est centrée sur la partie traitant de l'Italie. La tête de turc de la future école des Annales se voit (ibid., p. 158) accusée

4 Sur les relations de Pareto et de Ciccotti, voir Pareto, 1984. Voir aussi Busino, 1983, p. 14-15. Ciccotti (1863-1939) a donné, au lendemain de la mort de Pareto, un texte intéressant sur Pareto et les études historiques (voir la bibliographie du présent article). 
d'ignorer Guglielmo Ferrero. Mais une plus grave critique lui est adressée : «En général, les causes sociales des événements lui échappent». L’histoire pour Pareto n'est pas, en effet, «évènementielle»; mais elle est bien plus qu'«économique et sociale»: elle assigne aux représentations, aux sentiments et aux croyances une place déterminante dans le devenir historique. Le Traité de sociologie générale en administre la preuve.

\section{LES CONSIDÉRATIONS SUR L'HISTOIRE DANS LE TRAITÉ DE SOCIOLOGIE GÉNÉRALE}

Le Traité de Pareto est jalonné autant de références à des époques ou à des situations passées, que de réflexions sur l'histoire telle que les historiens l'écrivent et l'enseignent. Les unes sont au reste souvent inséparables des autres. On s'attachera ici aux jugements portés sur la démarche et la méthode historique, non aux exemples que l'histoire fournit. Ces derniers renvoient à des «faits», comme ceux qu'enregistrent ou consignent les annales, les chroniques, les coutumes; les premiers sont inspirés par l'interprétation qu'en donnent les historiens ; tous se distinguent par leur pertinence et leur tonalité souvent polémique. Le dernier chapitre (XIII), «L'équilibre dans l'histoire», est, comme son titre l'indique, particulièrement nourri de références à l'histoire ancienne, moderne et contemporaine. Il est ainsi d’entrée posé que «la Grèce antique fut un laboratoire d'expériences sociales et politiques, riche en observations très étendues 》 (\$2419). Le chapitre précédent «Forme générale de la société » est aussi lesté de matériaux historiques; il comprend, en outre, une suite de paragraphes (\$2156 et suiv.) consacrés à l'histoire comme discipline. Le Traité est cependant constellé de notations concernant l'«histoire» qui ne sont que partiellement récapitulées, sous ce terme, dans la table analytique des matières.

5 Sauf indications contraires, toutes les références bibliographiques qui suivent renvoient aux paragraphes du Traité de sociologie générale (Pareto, 1968 [1916]). 
Les «faits sociaux » sont les éléments que Pareto se propose d'étudier (§I44); ils forment la trame de l'histoire. La classification savante qu'il entreprend d'en faire et qui donne au Traité ses divisions en masque une autre, plus élémentaire, qui a son importance: dans l'ensemble des faits sont distinguées (§80) une «aristocratie», une «classe moyenne» et une «basse classe». La première est privilégiée, et la troisième dédaignée. Une partition noble/ignoble marque, comme on sait, l'histoire traditionnelle seulement attentive aux faits politiques, diplomatiques, militaires. Elle est refusée par Pareto: «Nous accueillerons tous les faits, quels qu'ils soient, pourvu que, directement ou indirectement, ils puissent nous conduire à la découverte d’une uniformité» (§8I). Des faits qui peuvent paraître «insignifiants ou puérils » seront pris en compte dès lors qu'ils peuvent permettre de connaître «les idées et les sentiments des hommes» (§82). L'opposition d'une grande et d'une petite histoire, l'une macroscopique, l'autre microscopique, n’a pas lieu d'être. ${ }^{6}$ Tout est bon à recueillir qui met en évidence certaines uniformités et «qu'il existe entre celles-ci des liens de mutuelles dépendance» (\$96). Les romans ne sont pas à négliger: s’y manifestent des opinions et des attentes caractéristiques d'une époque?

De façon similaire, Pareto critique les «théoriciens» qui généralement ne s’intéressent quaux actions logiques : divers motifs agissent «pour [les] éloigner du champ des actions non-logiques, et pour les refouler dans celui des actions logiques» (\$264). Il étend ce qu'il dit d'eux aux historiens : «Beaucoup d’historiens parlent des actions non logiques sans leur donner ce nom, et sans s'occuper d’en faire la théorie» (\$358). Cette tendance à ranger celles-ci parmi les préjugés

6 Pareto fait son miel des Mémoires et des Souvenirs. Ainsi, sur le Second Empire, outre la série de L'Empire libéral (1894-1902) d'Émile Ollivier, il utilise ceux de Henri-Joseph Dugué de la Fauconnerie, de Bernard-Adolphe Granier de Cassagnac, du très spirituel comte Horace de Viel Castel. Les ouvrages de E. Fournier et du Bibliophile Jacob (Paul Lacroix), grands érudits dont la curiosité d'esprit est dégagée de véritable préoccupation scientifique, font partie de sa documentation-comme le Journal des Goncourt.

7 Traité (\$545) «Beaucoup de romans nous font également connaître les opinions existantes; celles-ci correspondent souvent à certains faits, et en donnent une idée synthétique, meilleure que celle qu'on pourrait avoir de témoignages directs, nombreux et confus ». Les romans de Zola et de Maupassant sont cités en exemple (n.2). «Les Misérables de Victor Hugo et, si l'on veut, les romans de Georges Sand, donnent une idée précise et claire des débordements de l'humanitarisme dans les sociétés civilisées du XIXe siècle» (n.3). 
et à vouloir que toute action soit logique date du XVIII ${ }^{\mathrm{e}}$ siècle $(\$ 545, \mathrm{n} .3)$; elle est lourde de conséquences: l'interprétation logique d’actions non-logiques est source de biais et d'erreurs, singulièrement s'agissant des croyances. Le parti pris rationaliste de Voltaire fournit un bon exemple: il éclate dans un texte sur la religion romaine où «les erreurs historiques abondent» (§3ı n. n). Cela conduit à une réévaluation et à une réhabilitation de legs du passé disqualifiés par les philosophes des Lumières - fables, superstitions, traditions populaires : elles nous renseignent, pour une époque donnée, sur les «sentiments» éprouvés, la sensibilité collective, tout ce qui fait aujourd'hui l'objet de l'histoire des «mentalités».

Le Traité ouvre une troisième brèche, au voisinage de la précédente, dans l'armature de l'histoire traditionnelle. «D’une façon générale, on peut dire que jusqu'à présent, on a fait l'histoire des dérivations plutôt que celle des résidus » (§2156). En choisissant d'étudier des conceptions plutôt que «les forces manifestées par ces conceptions », on opte pour un type d'histoire qui n’a pas pour unique but de «décrire les faits réels et leurs rapports» (\$2157). On retrouve ici le thème fondamental que les sentiments et leurs diverses manifestations sont des «faits» aussi importants que les actions (\$54I). Une partie de celles-ci (les actions nonlogiques) résultent d'«un certain état psychique: sentiments, subconscient, etc. ». La psychologie s'en occupe, et Pareto nous prévient que, dans son étude, il part de «cet état de fait, sans vouloir remonter plus haut» (§I6I). La porte est donc fermée à une histoire psychanalytique centrée sur l'exploration de l'inconscient collectif $^{8}$, mais non à une psychologie historique-celle que Alphonse Dupront devait conceptualiser et pratiquer-, en prise sur les mentalités.

$\mathrm{Au}$ fil de ces considérations, les éléments d’une méthodologie historique sont présentés. Celle-ci a pour fondement les «faits», hors desquels on tombe dans le roman. On le constate avec le second Discours de Rousseau Sur l'origine et les fondements de l'inégalité parmi les hommes (1754). La parole inaugurale

8 La conjoncture intellectuelle dans laquelle se fit, en 1968, la réédition du Traité, par les soins de Busino, fut propice à ce type d'interprétation de la pensée parétienne. II est ainsi annoncé, en quatrième de couverture, que «Comme Marx et Freud, Pareto, bien que partant de points différent et ne visant aucun but concret, nous a montré un processus d'exploration de l'inconscient collectif. II est indubitable qu'il l'a fait sans aucun respect de la raison, avec passion et violence polémique». 
«Commençons donc d'abord par écarter tous les faits, car ils ne touchent point à la question » apparaît dénuée de sens à Pareto. D'aucuns objecteront qu'il s'agit là d'un modèle dont l'idée lui échappe, et non d'un roman des origines. Il n’en est pas moins ironiquement conclu qu'«il est beau d'écrire ainsi l'histoire, et de tirer d'hypothèses tout à fait incertaines, la description de temps tout à fait inconnus» (\$822). Ce genre de spéculations est sans valeur. Pour qu’un raisonnement soit acceptable, il doit se soutenir d'une assise documentaire. À l'égyptologue Jacques de Morgan, est empruntée la nomenclature des documents «sur lesquels s'appuie l'histoire proprement dite». Extraite des Premières civilisations-Études sur la préhistoire et l'histoire jusqu'à la fin de l'empire macédonien (1909), elle est résumée et reproduite en note du paragraphe 536 intitulé «Les faits ». Une série d’observations et de prescriptions est ensuite déroulée (\$537-552).

«Il vaut mieux, beaucoup mieux, citer trop de faits que de n'en citer aucun $»^{9}$ (\$54I). Il faut les sélectionner et les agencer avec prudence, ne retenir que les plus certains, sans viser la précision absolue. On ne se perdra pas dans les détails; une prise de vue préalable est nécessaire: elle est un moment de la méthode des approximations successives. Les textes venant au premier rang de la documentation à rassembler, les niveaux, sinon les règles, de leur étude sont formulés : «On peut rechercher I) ce que pensait l’auteur, quel était son état psychique, et comment il a été déterminé ; 2) ce que signifie un passage déterminé ; 3) comment l'ont compris les hommes d’une collectivité donnée en un temps déterminé » (ibid.). La démarche et codifiée, qui doit donner de bons résultats : «Soit $A$, un fait du passé dont nous ignorons l'“explication”. Nous l'obtenons au moyen d'une certaine interprétation, c'est-à-dire que nous mettons $A$ en rapport avec un autre fait B. Ensuite nous devons rechercher si ce genre d'interprétation conduit ou non à des résultats probables », etc., (\$547). Cet exposé s’achève sur des considérations intéressant «la probabilité des conclusions ».

9 Cette affirmation est assortie d'une remarque piquante concernant «l'usage pédant des "bibliographies compètes" aujourd'hui à la mode. Qui traite un sujet doit citer tous les auteurs en la matière» (\$538). Voir aussi ce qui est dit des citations (\$648): autrefois il n'y en avait presque jamais, l'auteur ne s'en souciait pas, on devait s'en remettre à son autorité, «mais aujourd'hui, dans les ouvrages historiques, l'usage général est différent». 
Le rôle du présent dans l'explication du passé est précisé : «On doit en général expliquer l'inconnu par le connu; c’est pourquoi le passé s'explique mieux par le présent, que le présent par le passé » (\$548); «La possibilité de faire des vérifications directes, dans le sens que nous pouvons faire de nouvelles observations, est aussi un motif pour expliquer les faits du passé » (\$57I). À toutes les étapes, la vérification, le contrôle s’imposent, tout comme la libre discussion des thèses proposées. Une remarque, qui fait penser à l'épistémologie poppérienne des sciences, est introduite par Pareto : «pour juger de la vérité d'une théorie», il est «indispensable qu'on puisse la combattre librement. Toute restriction $[\ldots]$ mise à qui veut la contredire, suffit pour en faire douter» (\$568). Dans les explications données, l’imputation causale doit être, par ailleurs, révisée. C'est ainsi à tort que Fustel de Coulanges a placé, dans La Cité antique, la religion et la propriété dans un rapport de cause à effet, en soutenant que c'est la religion domestique qui a conduit l'homme à s'approprier la terre (\$254). Dans ce cas particulier, est mise en évidence une « erreur très générale qui consiste à substituer des relations de cause à effet à des relations de mutuelle dépendance» (\$255).

On sait qu’avec l'hétérogénéité sociale, la mutuelle dépendance des phénomènes sociaux est l'un des thèmes majeurs de la sociologie de Pareto. L'histoire en manifeste l'effectivité. Une critique du matérialisme historique en découle : cette doctrine a certes été un progrès scientifique, en ce qu'elle a fait apparaître comme contingent des phénomènes d'ordre idéologique auxquels on conférait un caractère absolu; elle a bien mis en lumière la mutuelle dépendance des phénomènes économiques et sociaux, mais son erreur est de l’avoir remplacée par une relation de cause à effet (voir §829)..$^{\circ}$ Dix ans plus tôt, dans la recension précédemment mentionnée de l’ouvrage de Ciccotti, la critique de «l'interprétation matérialiste de l'histoire » était déjà esquissée. Il est possible d'expliquer l'histoire soit par les idéologies, soit par les rapports économiques et sociaux. S’il fallait faire un choix entre ces deux «hypothèses», il faudrait opter pour

I0 L'autre erreur du matérialisme historique est d'expliquer tous les faits que rapporte l'histoire par une cause unique : l'exploitation du prolétariat par la bourgeoisie, «et subsidiairement la résistance du prolétariat à l'exploitation» (\$830). 
la seconde car «elle se rapproche plus de la vérité». Mais, ajoute Pareto, «le dilemme n'existe pas». Il y a «entre les phénomènes d'autres rapports que ceux de causes à effets $[\ldots]$ c'est-à-dire des rapports de mutuelle dépendance » (1966a, p. I26). Il reviendra encore sur cette question dans «Le phénomène bolchévique » : «Nous remarquons dans l'histoire une extraordinaire concordance entre les événements et [...] les idées ». Dire que les événements sont la cause des idées est moins inexact que de soutenir l'inverse; mais «tout ce qui est connu s'explique beaucoup mieux si l'on accepte que les événements et les idées sont interdépendants 》 (1976, p. I88).

Ce qui biaise ou barre l'intelligence de l'histoire est souvent signalé. Maintes fois (par exemple aux paragraphes 552 et 24I du Traité) est dénoncée l'«intromission» des sentiments, de la morale et des intérêts dans l'analyse scientifique; elle peut être chez l'historien, comme chez tous les auteurs, plus ou moins consciente, plus ou moins volontaire. Elle est en tout cas dommageable en ce qu'elle altère le sens de ce qui s'est «réellement» passé. De même les voiles dont les historiens enveloppent leurs récits empêchent de voir les «faits réels»; il est impératif de les ôter (\$2357). Les pièges, depuis appelés «ethnocentrisme» et «européocentrisme», sont bien aperçus: «Les plus grandes difficultés que nous éprouvons à comprendre les faits d’autres peuples ou d'autres temps, proviennent de ce que nous les jugeons avec les habitudes mentales de notre nation ou de notre temps» (\$55 I). L'érudition historique permet de les éviter : «viv(ant) en partie dans le passé», ceux qui la possèdent sont ici en meilleure posture: ils comprennent «les faits mieux que les profanes» (ibid.). Les difficultés que rencontre tout changement dans la représentation du passé sont latéralement mises en évidence. Niebuhr, Mommsen, Grote et bien d'autres ont montré «la vanité historique des légendes antiques. [Ils] en ont définitivement débarrassé l'histoire» (\$656). Mais «Les hommes ne se résignent pas volontiers à abandonner leurs légendes» (\$657) ; la lenteur avec laquelle s'opère le remaniement de la mémoire collective est sous-entendue.

Associée à l'étude des rapports qu'entretiennent légendes et «faits réels», c'est-à-dire mythe et histoire, une double entreprise de démythification et de démystification est conduite dans le Traité. Le travail qui consiste à démêler ce qui correspond d'une part à des actions non-logiques, d'autre part à des revête- 
ments logiques ou pseudo-logiques (voir §798), à dévoiler aussi les résidus que recouvrent les dérivations, intéresse directement l'histoire. Ainsi, les historiens de la Révolution française «nous donnent un mélange de dissertations éthiques et [...] d'observations de faits » (\$2 I68). Alphonse Aulard et Hyppolite Taine sont renvoyés dos à dos, comme le sont admirateurs et détracteurs de la Révolution; en réalité, la même phraséologie destinée à agir sur le sentiment était employée par les partis qui s’affrontaient: «des dérivations identiques recouvr[aient] des résidus différents. C’est pourquoi celui qui s’arrête aux dérivations ne peut rien connaître des forces qui agissaient en réalité » (\$2169).

Deux sortes d'ouvrages historiques sont distingués à partir de l'exemple précédent : ceux dont les auteurs se soucient uniquement de l'accord avec les faits, ceux où divers «jugements éthiques sont prononcés». Ces derniers renvoient à des entités supérieures, mais vagues. Rares étaient autrefois les histoires qui ne faisaient pas intervenir les dieux pour expliquer les événements. Aujourd'hui on admet, plus ou moins explicitement, «que principes et théories donnent au phénomène social sa forme» (\$I765). De métaphysique l'histoire est devenue éthique. Une distribution plus originale est exposée au paragraphe 1580: sont distingués l'histoire scientifique (description des faits et de leurs rapports), le roman historique (de «lecture agréable»), et des «compositions flottant entre l'histoire scientifique et le roman historique». L’histoire est par là pensée par Pareto en termes d'activité scientifique, de fonction onirique et d'usage social. Nombre d'histoires contemporaines donnent dans le mélange des genres, et leurs auteurs ont «recours à l'amphibologie du terme vérité historique» pour le dissimuler (\$2I6I). Avec cet ajout de vues éthiques et de propos esthétiques «on prétend faire une histoire "vivante”, par opposition à l'histoire "morte” qui serait celle qui vise exclusivement à être d’accord avec les faits » (§663).

Au total, la méthode «historique» présente un intérêt et des limites qui sont clairement dégagés. Il lui revient d’avoir «ouvert la porte par laquelle l'expérience s'est introduite dans une partie des sciences sociales» (§6I9). Mais elle ne doit pas être confondue avec la méthode expérimentale. Propre à manifester les contingences de temps et de lieu, utile pour connaître la succession des faits, elle néglige les rapports qui existent entre eux et échoue à montrer 
leur mutuelle dépendance. Aussi bien, l'abus que l'on fait maintenant de cette méthode, particulièrement dans les sciences économiques et sociales, est-il préjudiciable à celles-ci (\$857). Une conception "structurale” de l'histoire se profile ainsi, donnant plus d'importance, dans l'étude des phénomènes, à l'ordre synchronique qu'à la dimension diachronique. Elle est sous-jacente à l'examen d'uniformités auquel se livre l'auteur du Traité. Par exemple, la forme périodique, oscillatoire, ondulée des phénomènes naturels et sociaux a donné lieu à quantité de théories. Faire l'étude historique de ces dernières n’est d'aucune utilité, «n’en déplaise aux fanatiques de la "méthode historique" et aux partisans des "bibliographies complètes" » (\$2330); mieux vaut recourir à des témoignages directs et rechercher des indices mesurables en vue d'une classification. C’est ce que Pareto s'est employé à faire au §2293, en distinguant, à partir d'un cas précis, temps conjoncturel et longue durée, à savoir trois types de variations : accidentelles, à courte période, à longue période ${ }^{11}$.

La comparaison faite, au paragraphe 24I0, des deux expressions «l'histoire se répète toujours», «l'histoire ne se répète jamais » éclaire finalement la conception que Pareto s'est formée de l'histoire-et l'utilisation qu'il en fait. La répétition ne s'opère pas à l'identique, de la même façon factuelle; mais elle se vérifie «en certaines parties que nous pouvons dire principales». Ces parties donnent ses paramètres au modèle du changement social exposé dans le Manuel (chap. II) et notablement enrichi dans le Traité. Montage d'événements historiques et de faits directement observés, il montre «dans toute l'histoire» l'alternance de la force et du consentement comme moyens de gouvernement; que l'histoire des sociétés humaines est principalement celle de la succession des aristocraties; qu'il y a concordance entre type de résidus et «forme générale de la société ». Il fait voir les conséquences qu’entraînent les modifications

II Pour ces dernières - les variations à longue période - Pareto note qu'«elles n'ont pas été étudiées jusqu'à présent, cela en grande partie parce qu'on avait pas encore les données statistiques nécessaires» (\$2293). Elles l'ont été depuis (voir par exemple les fluctuations de longue durée analysées par Nicolaï.D. Kondratieff). D'une manière général, est-il indiqué dans le Traité, «les sciences tendent à devenir quantitatives» (\$|44); en histoire aussi les études quantitatives seront, en partie, substituées aux considérations qualitatives (songeons à l' «histoire sérielle» de Pierre Chaunu). 
des proportions des deux premières classes de résidus dans l'élite au pouvoir et la classe gouvernante. Ce modèle, centré sur le devenir des élites et leur circulation, permet d'expliquer aussi bien le conflit d'Athènes (où prime l'instinct des combinaisons) et de Sparte (où domine la persistance des agrégats), que l'antagonisme entre la France de Napoléon III, où s'activent les affairistes de la fête impériale, et la Prusse de Guillaume Ir forte d'une puissante noblesse cultivant les vertus militaires. Partout et toujours on enregistre l'opposition des Spéculateurs (déracinés) et des Rentiers (enracinés). Mais, prévient Pareto (\$24I4 et §24I5) ces proportions et leurs variations ne doivent pas être prises comme causes ou conditions nécessaires ou suffisantes. Elles doivent être mises en relation avec d'autres phénomènes sociaux.

Tels sont, schématiquement indiqués, les principaux éléments constitutifs de la sociologie historique de Pareto. Celle-ci est résolument comparative. Les superlatifs pour qualifier l'étendue des connaissances en histoire qu'elle intègre ne manquent pas; on dira simplement que ce savoir est d'une exceptionnelle richesse. L'usage qui en est fait dans le Traité est marqué au coin du génie, mais il n’est pas toujours équitable. Ainsi, dans le douzième chapitre (\$2330), à propos des oscillations, et après avoir dit de la théorie de Vico sur les Corsi e ricorsi qu'elle est «principalement métaphysique», il en vient à examiner la Théorie des périodes politiques (I874) de Giuseppe Ferrari. Cet ouvrage «paraît nous conduire en plein domaine expérimental»; il n'en est cependant rien, car «l'auteur traite un peu trop arbitrairement les faits », et les longs commentaires qui suivent mêlent de façon alambiquée compliments et critiques. La pensée de Ferrari, qui a publié plusieurs livres intéressants sur Vico, est cependant riche d’aperçus très originaux - ce dont d’ailleurs ne disconvient pas Pareto. Mais seul compte l'«accord avec les faits ». Là encore, et comme tout au long de l'œuvre de ce dernier, se fait entendre la rituelle invocation de «faits», tenus pour « réels», comme s'ils n'étaient pas des constructions ressortissant parfois, en particulier dans les sciences humaines et sociales, à la fiction. 


\section{BIBLIOGRAPHIE}

BUSINO G., 1968, Introduction à une histoire de la sociologie de Pareto, Genève, Droz.

-, 1983, Pareto, Croce, les socialismes et la sociologie, Genève, Droz.

CICCOTTI E., 1924, «Pareto e gli studi sorici», Giornale degli Economisti e Rivista de Statistica, 39-I, p. ||4-||9.

FEMIA J.V., MARSCHALL A.J., 20I2, Vilfredo Pareto Beyond Disciplinary Boundaries, Farnham, Ashgate.

MONGARDINI C., 1965, «Mosca, Pareto e Taine», Cahiers Vilfredo Pareto/Revue européenne d'histoire des sciences sociales, 5, p. 175-186.

PARETO V., 1964 (I896-1897), Cours d'Économie politique, Euvres complètes, t. I-II, Genève, Droz.

-, 1965a, Libre-échangisme, protectionnisme et socialisme, Fuvres complètes, t.IV, Genève, Droz.

-, 1965b (1901-1902), Les Systèmes socialistes, Fuvres complètes, t.V, Genève, Droz.

-, 1966a , Mythes et idéologies, Fuvres complètes, t. VI, Genève, Droz.

-, 1966b (1906), Manuel d'Économie politique, CEuvres complètes, t. VII, Genève, Droz.

-, 1968 (1916), Traité de sociologie générale, Euvres complètes, t.XII, Genève, Droz.

-, 1975, Jubilé du Professeur V. Pareto, Fuvres complètes, t.XX, Genève, Droz.

-, 1976, Faits et théories, Euvres complètes, t.XXI, Genève, Droz.

-, 1984, Lettere a Maffeo Pantaleoni (1890-1923), Fuvres complètes, t.XXVIII, Genève, Droz.

TOMMISSEN P., BUSINO G., 1975, «Bibliographie des écrits de et sur Vilfredo Pareto», in Vilfredo Pareto, Fuvres complètes, t. XX, Genève, Droz, p.7I-249.

VALADE B., 197I, «Pareto historien de l'Europe contemporaine», Ethnopsychologie, 4, p.449-466.

-, 2006, «Du bon usage de l'histoire selon Pareto », in Peut-on faire confiance aux historien? J. Tulard (éd.), Paris, PUF, p. 55-70. 
